\title{
AN ALGEBRAIC THEORY OF LOCAL KNOTTEDNESS. I
}

\author{
BY \\ SAMUEL J. LOMONACO, JR.(1)
}

\section{0 . Introduction.}

A. Summary. A significant problem in topology is the classification of wild arcs in $S^{3}$. The first fundamental paper in this area was written by Fox and Artin in 1948 [13]. There the authors developed rigorous algebraic means for demonstrating the nontameness of arcs. Some time later (1962) Fox and Harrold [14] succeeded in completely classifying a special class of wild arcs, the Wilder arcs. In 1960 Brody [6] developed invariants of infinitely generated modules and used such invariants to distinguish wild knots. Finally, in 1962 ([1], [2]) Alford and Ball constructed a geometric invariant, the penetration index, capable of distinguishing a large class of wild arcs.

In this paper we develop algebraic means for distinguishing wild arcs in $S^{3}$ which go significantly beyond previously known methods. Unlike most of the above authors we consider the problem from a local rather than a global standpoint.

The first three sections of this paper are devoted to extending and refining Brody's algebraic techniques [6].

In $\S I V$, a fundamental invariance theorem (IV.B.3) is proven, i.e., (roughly) if $p$ is an isolated singular interior point of an arc $k$ and $U$ is an arbitrary suitably nice neighborhood of $p$, then the fundamental group $\Pi(U-k)$ of $U-k$ modulo an equivalence relation, called local equivalence, is an invariant of the singularity $p$. Thus, the invariants of $\Pi(U-k)$ modulo local equivalence, i.e., the module $\mathfrak{M}$ (associated with $\Pi(U-k)$ ) modulo an equivalence relation (III.B.2, III.B.7, IV.A.3, IV.C.1), the $k$ th divisor chains of $\Pi(U-k)$ (II.A.4, III.C.3, IV.C.2), the $k$ th local topologies $\Lambda_{n}(k, p)$ of $\Pi(U-k)$ (I.D.3, II.A.1, II.A.5), etc., also become invariants of the singularity.

In $\S \mathrm{V}$ some applications are considered. First (V.A) the algebraic invariants of Wilder arcs are found to have some pleasant properties (V.A.5). Moreover, the algebraic analogue (V.A.6) to the geometric classification of Wilder arcs [14] is

Presented to the Society, January 29, 1965; received by the editors October 11, 1965.

(1) The results in this paper are from my doctoral dissertation, submitted to Princeton University in September, 1964. I am especially grateful to my dissertation advisor Professor Ralph H. Fox for suggesting the topic from which this paper evolved and for his more than helpful direction and suggestions. I am grateful to Professors C. Papakyriakopoulos, J. Stallings, and $\mathrm{H}$. Trotter for some very stimulating and rewarding conversations. I would also like to thank N. Smythe and C. Giffen for their suggestions and criticisms.

This work was supported in part by National Science Foundation Grant GP-5458. 
presented. Next (V.B, V.C) two different types of compositions of arcs are considered (each analogous to the composition of tame knots). The algebraic invariants of such composites are shown (V.B.5, V.C.10, V.C.11) to be computable from the algebraic invariants of their factors. In studying such arc composites, we find (V.B.7, V.C.12, V.C.13) that there is very little relation between the algebraic invariants of this paper and the penetration index of Alford and Ball ([1], [2]). Finally in V.D., the algebraic invariants of a number of examples are computed.

B. Preliminaries. Before proceeding to the main body of this paper, a few preliminary definitions are necessary.

Definition. Let $k$ and $k^{\prime}$ be oriented arcs in $S^{3}$ and let $p$ and $p^{\prime}$ be points of $k$ and $k^{\prime}$ respectively. $k$ at $p$ is of the same local type as $k^{\prime}$ at $p^{\prime}$ if there are open neighborhoods $U$ of $p$ and $U^{\prime}$ of $p^{\prime}$ and an orientation preserving homeomorphism of $U$ onto $U^{\prime}$ (where $U$ and $U^{\prime}$ each inherit their orientation from that of $S^{3}$ ) that maps $(U \cap k, p)$ onto $\left(U \cap k^{\prime}, p^{\prime}\right)$.

Definition. Let $p$ be a point of an arc $k$ in $S^{3} . k$ is locally tame at $p$ if there exists a neighborhood $U$ of $p$ and a homeomorphism $h_{p}$ of the closure $\bar{U}$ of $U$ into $S^{3}$ such that $h_{p}(\bar{U} \cap k)$ is a polyhedron ([4, p. 146]). If $k$ is locally tame at $p$, then $p$ is a tame point of $k$. If not, $p$ is a wild point of $k$. A wild point $p$ of $k$ is a singular point if there exists a neighborhood of $p$ containing no other wild point of $k$.

Definition. $k$ at $p$ is of the trivial local type if $p$ is a tame point of $k$. Obviously, there are exactly two trivial local types, corresponding to terminal and interior points of arcs.

In terms of the above definitions the scope of this paper is restricted to the development of algebraic invariants of the local types of oriented arcs at singular or tame interior points.

For purposes of simplification the following two conventions are made. First, only arcs with at most one wild point will be considered. This entails no loss in generality; for if $p$ is a singular point of an arc $k$, then there is a subarc $k^{\prime}$ of $k$ of the same local type at $p$ having $p$ as its only wild point. Second, all results are stated for singular points of arcs, although they are obviously also valid for tame points.

I. Ideal topologies. In this and the next two sections Brody's algebraic theory [6] is extended. Throughout these sections $R$ will denote a countable Noetherian unique factorization domain. Since all proofs in this section are of a straightforward nature, they have been omitted.

A. Introduction.

1. Definition. A topology $I$ on $R$ is an ideal topology if there exists a collection of ideals $\left\{I_{\alpha}\right\}$ such that the collection $\left\{a+I_{\alpha}: a \in R, I_{\alpha} \in\left\{I_{\alpha}\right\}\right\}$ forms a basis for the open sets of $I$.

2. Remark. Obviously, every ideal neighborhood basis about zero for an ideal topology uniquely determines that topology. 
3. Remark. $R$ together with an ideal topology forms a topological ring.

4. If $\left\{I_{\alpha}\right\}$ is a collection of ideals of $R$ such that the intersection of any two members of this collection contains a third, then $\left\{I_{\alpha}\right\}$ may be considered as a basis of neighborhoods about zero of an ideal topology.

B. The calculus of ideal topologies. For notation not defined herein please refer to $[6, \S 1]$.

Let $\left\{\boldsymbol{I}_{\alpha}\right\}$ be a family of ideal topologies and for each $\alpha$ let $\left\{I_{\beta \alpha}\right\}$ be an ideal basis of neighborhoods about zero for $\boldsymbol{I}_{\alpha}$.

1. Definition. The supremum of the family $\left\{\boldsymbol{I}_{\alpha}\right\}$, written $\bigcap \boldsymbol{I}_{\alpha}$, is the coarsest ideal topology finer than all $\boldsymbol{I}_{\alpha}$, i.e., the supremum with respect to the ordering $\dot{\succ}$.

It can easily be verified that the collection of all finite intersections of the ideals $\left\{I_{\beta \alpha}\right\}$ is an ideal neighborhood basis about zero for $\bigcap \boldsymbol{I}_{\alpha}$; hence, the notation.

2. Definition. The product of the family $\left\{\boldsymbol{I}_{\alpha}\right\}$, written $\prod \boldsymbol{I}_{\alpha}$, or as $\boldsymbol{I}_{1} \cdot \boldsymbol{I}_{2} \cdots \boldsymbol{I}_{n}$ in case the family is finite, is the ideal topology having as an ideal neighborhood basis about zero the collection of all finite products $P_{y}$ of the ideals $\left\{I_{\beta \alpha}\right\}$ such that each factor $I_{\beta \alpha}$ of $P_{\gamma}$ is from a differently indexed ideal topology.

3. Definition. The sum of the family $\left\{\boldsymbol{I}_{\alpha}\right\}$, written $\sum \boldsymbol{I}_{\alpha}$ or $\boldsymbol{I}_{1}+\boldsymbol{I}_{2}+\cdots+\boldsymbol{I}_{n}$ in case the family is finite, is the finest ideal topology coarser than all $\boldsymbol{I}_{\alpha}$, i.e., the infimum with respect to the ordering $>$.

It readily follows that the collection of all ideals of the form $\sum I_{\alpha}, I_{\alpha} \in\left\{I_{\beta \alpha}\right\}_{\beta}$, summed over all $\alpha$, is an ideal neighborhood basis about zero for $\sum \boldsymbol{I}_{\alpha}$; hence, the notation.

C. Principal ideal topologies.

1. Definition. A principal ideal topology is an ideal topology having a collection of principal ideals as one of its ideal neighborhood bases about zero.

2. Notation. Let $p$ be an element of $R$. Henceforth, $p$ will also denote the principal ideal topology having $\{(p)\}$ as an ideal neighborhood basis about zero, where $(p)$ denotes the ideal generated by $p$. Hence, $\left\{\left(p^{n}\right)\right\}$ is an ideal basis of $p^{n}=p \cdot p \cdot \cdots \cdot p$ (n factors) and $\left\{(p),\left(p^{2}\right),\left(p^{3}\right), \ldots\right\}$ is an ideal neighborhood basis of $p^{\infty}=p \cdot p \cdot p \cdot \cdots$.

3. Definition. Let $I$ be an ideal topology. An irreducible element $p$ of $R$ is a prime factor of $I$ if $(p)$ is open in $I$. If $p$ is an element of $R$, then the exponent of $p$ with respect to $I$, written $e(p, I)$, is the largest nonnegative integer $n$ such that $\left(p^{n}\right)$ is open in $I$. If no such largest nonnegative integer exists, then $e(p, I)=\infty$.

4. Let $\left\{p_{n}\right\}$ be an indexing on the positive integers of all distinct (up to associates) primes of $R$. Then every principal ideal topology I may be factored uniquely into the form $\prod p_{n^{n}}^{e}$ where $e_{n}$ is a nonnegative integer or $\infty$. Moreover, $e_{n}=e\left(p_{n}, I\right)$.

5. If $I$ and $I^{\prime}$ are principal ideal topologies such that $I>I^{\prime}$ and if $p$ is an irreducible element of $R$, then $e(p, I) \geq e\left(p, I^{\prime}\right)$.

6. Definition. The principal sum of a family of ideal topologies $\left\{\boldsymbol{I}_{\alpha}\right\}$, written $\sum^{*} I_{\alpha}$, or $I_{1}+{ }^{*} I_{2}+{ }^{*} I_{3}+{ }^{*} \ldots+{ }^{*} I_{n}$ in case the family is finite, is the coarsest principal ideal topology finer than $\sum I_{\alpha}$. 
D. Ideal topology chains.

1. Definition. An ideal topology chain is a monotone decreasing sequence of ideal topologies.

2. Definition. Two ideal topology chains $I_{n}$ and $I_{n}^{\prime}$ are equivalent if

(a) for every $n$ there exists an $m(n)$ such that $I_{n} \succ I_{m(n)}^{\prime}$,

(b) for every $m$ there exists an $n(m)$ such that $I_{m}^{\prime}>I_{n(m)}$.

3. If $\boldsymbol{I}_{n}$ and $\boldsymbol{I}_{n}^{\prime}$ are two equivalent ideal topology chains, then $\sum^{*} \boldsymbol{I}_{n}=\sum^{*} \boldsymbol{I}_{n}^{\prime}$.

4. Definition. Ideal topologies $I$ and $I^{\prime}$ are similar if there exists $p$ and $p^{\prime}$ in $R$ such that $p I>I^{\prime}$ and $I \prec p^{\prime} I^{\prime}$.

5. Two principal ideal topologies $I$ and $I^{\prime}$ are similar if and only if:

(a) I and $I^{\prime}$ have the same set of prime factors with infinite exponent, and

(b) $e(p, I)=e\left(p, I^{\prime}\right)$ for almost all (up to associate) irreducible elements $p$ of $R$.

6. Definition. An ideal topology chain $\boldsymbol{I}_{n}$ is comparable if there exists a positive integer $N$ such that for every $m, n \geqq N, \boldsymbol{I}_{m}$ and $\boldsymbol{I}_{n}$ are similar. Obviously, if $\boldsymbol{I}_{n}$ is comparable, then there exists an ideal topology $\boldsymbol{I}_{\infty}$ such that $\boldsymbol{I}_{n}$ is similar to $\boldsymbol{I}_{\infty}$ for almost all $n$. Any such ideal topology $I_{\infty}$ is called a comparing ideal topology of the chain $I_{n}$. It is immediate that any two comparing topologies are similar.

It now easily follows that comparability and the comparing ideal topology modulo similarity are invariants of equivalence.

II. Topologies of countably generated modules. In [6] Brody defined the $k$ th divisor topology $\nabla_{k}(\mathfrak{M})$ and the $k$ th residual topology $\Gamma_{k}(\mathfrak{M})$ of an arbitrary module $\mathfrak{M}$ for $k \geqq 0$. We now investigate these topologies only for countably generated (abbreviated c.g.) modules.

A. Local topologies and divisor chains. The author was not able to determine whether or not the residual topologies are principal ideal or even ideal topologies. To circumvent this difficulty the following definition is made.

1. Definition. Let $\mathfrak{M}$ be a c.g. $R$-module and $\left\{\mathfrak{M}_{\alpha}\right\}$ the collection of all finitely generated (abbreviated f.g.) submodules of $\mathfrak{M}$. The $k$ th local topology $\Lambda_{k}(\mathfrak{M})$ of $\mathfrak{M}$ is defined as: $\Lambda_{k}(\mathfrak{M})=\sum_{\alpha}^{*} \nabla_{k}\left(\mathfrak{M} / \mathfrak{M}_{\alpha}\right)$, i.e., $\Lambda_{k}(\mathfrak{M})$ is the finest principal ideal topology coarser than $\Gamma_{k}(\mathfrak{M})$.

The local topologies behave basically like the residual ones.

2. If $\mathfrak{M}$ is a c.g. R-module, then $\Lambda_{0}(\mathfrak{M}) \succ \Lambda_{1}(\mathfrak{M}) \succ \cdots$.

3. If $\mathfrak{M}$ is a homomorphic image of a c.g. R-module $\mathfrak{R}$, then $\Lambda_{k}(\mathfrak{R})>\Lambda_{k}(\mathfrak{M})$, for every $k \geqq 0$.

4. Definition. Let $\mathfrak{M}$ be a c.g. $R$-module and $\mathfrak{M}_{n}$ an arbitrary monotone increasing sequence of $\mathrm{f}$.g. submodules of $\mathfrak{M}$ whose union is $\mathfrak{M}$. A $k$ th divisor chain of $\mathfrak{M}$ is defined as the descending chain:

$$
\nabla_{k}\left(\mathfrak{M} / \mathfrak{M}_{1}\right) \succ \nabla_{k}\left(\mathfrak{M} / \mathfrak{M}_{2}\right) \succ \nabla_{k}\left(\mathfrak{M} / \mathfrak{M}_{3}\right) \succ \cdots .
$$

If Definition I.D. 2 is recalled, it may easily be shown that divisor chains modulo equivalence are invariants of c.g. modules: The divisor chains are related to the local topologies as follows: 
5. The principal sum of any kth divisor chain of a c.g. module $\mathfrak{M}$ is the kth local topology of $\mathfrak{M}$, i.e., $\Lambda_{k}(\mathfrak{M})=\sum_{n}^{*} \nabla_{k}\left(\mathfrak{M} / \mathfrak{M}_{n}\right)$.

B. Direct sums of modules.

1. If $\mathfrak{M}$ and $\mathfrak{R}$ are f.g. $R$-modules, then

$$
\Delta_{k}(\mathfrak{M} \oplus \mathfrak{R})=\sum_{i+j=k}^{*} \Delta_{i}(\mathfrak{M}) \Delta_{j}(\mathfrak{R}), \quad \text { for every } k \geqq 0,
$$

where " $\Sigma^{*}$ " denotes the smallest principal ideal containing the given collection of ideals.

Proof. Let $k \geqq 0$ be fixed and let $A$ and $B$ be relation matrices of $\mathfrak{M}$ and $\mathfrak{N}$ respectively. Let $m$ and $n$ be respectively the number of columns of $A$ and $B$ and let $\left\{A_{i}^{\alpha}\right\}$ and $\left\{B_{j}^{\beta}\right\}$ be the collection of all $(m-i) \times(m-i)$ and all $(n-j) \times(n-j)$ submatrices of $A$ and $B$, respectively $(i, j \geq 0)$.

It may be observed that any nonsingular submatrix of

$$
\left[\begin{array}{ll}
A & 0 \\
0 & B
\end{array}\right] \text { is of the form }\left[\begin{array}{cc}
A^{*} & 0 \\
0 & B^{*}
\end{array}\right],
$$

where $A^{*}$ and $B^{*}$ are nonsingular. Thus, $\Delta_{k}(\mathfrak{R} \oplus \mathfrak{R})$ is the smallest principal ideal containing all the elements $\left(\operatorname{det} A_{i}^{\alpha}\right)\left(\operatorname{det} B_{j}^{\beta}\right)$ where $i+j=k$, and $i j \geqq 0$. Q.E.D.

2. If $\mathfrak{M}$ is a c.g. R-module and $\left\{\mathfrak{M}_{\alpha}\right\}$ is a collection of f.g. submodules of $\mathfrak{M}$ such that:

(a) $\mathfrak{M}$ is generated by the union of the collection,

(b) The union of any two modules of the collection is contained in a third, then $\left\{\Delta_{k}\left(\mathfrak{M}_{\alpha}\right)\right\}$ is an ideal neighborhood basis about zero for $\nabla_{k}(\mathfrak{M})$, for every $k \geqq 0$.

3. If $\mathfrak{M}$ and $\mathfrak{R}$ are c.g. R-modules, then $\nabla_{k}(\mathfrak{M} \oplus \mathfrak{R})=\sum_{i+j=k}^{*} \nabla_{i}(\mathfrak{M}) \nabla_{j}(\mathfrak{R})$, for every $k \geqq 0$.

4. If $\mathfrak{M}$ is a c.g. $R$-module and $\left\{\mathfrak{M}_{\alpha}\right\}$ is a collection of f.g. submodules of $\mathfrak{M}$ satisfying (a) and (b) of II.B.2, then $\Lambda_{k}(\mathfrak{M})=\sum_{\alpha}^{*} \nabla_{k}\left(\mathfrak{M} / \mathfrak{M}_{\alpha}\right)$, for every $k \geqq 0$.

5. If $\mathfrak{M}$ and $\mathfrak{N}$ are c.g. $R$-modules, then $\Lambda_{k}(\mathfrak{M} \oplus \mathfrak{R})=\sum_{i+j=k}^{*} \Lambda_{i}(\mathfrak{M}) \Lambda_{j}(\mathfrak{N})$, for every $k \geqq 0$.

Proof. Let $k \geqq 0$ be fixed. Let $\left\{\mathfrak{M}_{\alpha}\right\}$ and $\left\{\mathfrak{N}_{\beta}\right\}$ be the collections of all f.g. submodules of $\mathfrak{M}$ and $\mathfrak{R}$ respectively. Then $\Lambda_{k}(\mathfrak{M} \oplus \mathfrak{R})=\sum_{\alpha, \beta}^{*} \nabla_{k}\left[(\mathfrak{M} \oplus \mathfrak{R}) /\left(\mathfrak{R}_{\alpha} \oplus \mathfrak{N}_{\alpha}\right)\right]$. Employing II.B.3 and interchanging the order of principal summations, we have the desired result. Q.E.D.

6. If $\mathfrak{M}_{n}$ is a sequence of f.g. R-modules, then

$$
\nabla_{k}\left(\bigoplus_{n=1}^{\infty} \mathfrak{M}_{n}\right)=\bigcap_{m=1}^{\infty} \sum_{i_{1}+i_{2}+\cdots+i_{m}=k}^{*} \prod_{n=1}^{m} \nabla_{i_{m}}\left(\mathfrak{M}_{n}\right)
$$

where $\bigoplus_{n=1}^{\infty} \mathfrak{M}_{n}$ denotes the weak direct sum.

As corollaries we have:

7. $\nabla_{0}\left(\bigoplus_{n=1}^{\infty} \mathfrak{M}_{n}\right)=\prod_{n=1}^{\infty} \nabla_{0}\left(\mathfrak{M}_{n}\right)$ and $\Lambda_{0}\left(\bigoplus_{n=1}^{\infty} \mathfrak{M}_{n}\right)=\sum_{m}^{*} \prod_{n=m+1}^{\infty} \nabla_{0}\left(\mathfrak{M}_{n}\right)$. 
8. If $\mathfrak{M}_{n}$ is a sequence of $f . g$. R-modules and $p$ is an irreducible element of $R$, then $p$ is a prime factor of $\Lambda_{0}\left(\oplus_{n=1}^{\infty} \mathfrak{M}_{n}\right)$ if and only if the ideal generated by $p$ contains $\Delta_{0}\left(\mathfrak{M}_{n}\right)$ for infinitely many $n$. Moreover, the exponent of every prime factor of $\Lambda_{0}\left(\bigoplus_{n=1}^{\infty} \mathfrak{M}_{n}\right)$ is infinite.

C. Methods of computation. We now seek methods for computing divisor and local topologies. Computations of such invariants will essentially be reduced to combinatorial operations on the relation matrices of c.g. modules. We begin by considering Brody's [6] elementary transformations in the countable case.

1. Definition. The nonsymmetric collection of elementary transformations is the following:

(0) Permute the rows in any way, or permute the columns in any way.

(I) Adjoin any countable set of rows such that each new row is a finite linear combination of the original rows.

(II) Adjoin a countable set of rows $r_{n}$ and columns $c_{n}$ such that the intersection of $r_{n}$ and $c_{n}$ is the identity 1 of $R$, such that all other entries of $c_{n}$ are zero, and such that there are at most finitely many nonzero entries in $r_{n}$ and all such nonzero entries lie in the original columns of the matrix.

The proof of the following is essentially that of the Tietze transformation theorem found in [8].

2. TheOREM ([6, p. 145]). Two c.g. R-modules are isomorphic if and only if each relation matrix of one may be obtained from each relation matrix of the other by a finite sequence of nonsymmetric elementary transformations.

3. Definition. The symmetric collection of elementary transformations is the following:

(0) Permute rows in any way or permute columns in any way.

$\left(\mathrm{I}_{0}\right)$ Adjoin any countable set of zero rows.

( $\left.\mathrm{II}_{0}\right)$ Adjoin a countable set of rows $r_{n}$ and columns $c_{n}$ such that the intersection of $r_{n}$ and $c_{n}$ is the identity 1 of $R$ and all other entries of $r_{n}$ and $c_{n}$ are zero.

(III) Add to each row of a given set of the rows any finite linear combination of the rows in the complementary set.

(III*) Add to each column of a given subset of the columns a (possibly infinite) linear combination of the columns in the complementary set such that each column in the complementary set appears in only finitely many such linear combinations.

The above set of elementary transformations is easily shown to be equivalent to the set of transformations found in II.C.1 [11].

From II.B. 2 the computation of a divisor topology of a c.g. module $\mathfrak{M}$ may be reduced to finding the elementary divisors of the terms of a monotone increasing sequence of f.g. submodules $\mathfrak{M}_{n}$ of $\mathfrak{M}$ whose union is $\mathfrak{M}$. The following indicates how such a sequence may be extracted from a particular relation matrix of $\mathfrak{M}$.

4. Let $\mathfrak{M}$ be a c.g. $R$-module and $j(1) \leqq j(2) \leqq \cdots$ an arbitrary monotone sequence of positive integers. Then there exists a montone sequence $i(1) \leqq i(2) \leqq \cdots$ of positive 
integers and a relation matrix of $\mathfrak{M}$ such that the first $i(n)$ rows and $j(n)$ columns constitute a relation matrix for a f.g. submodule $\mathfrak{M}_{n}$ of $\mathfrak{M}$ generated by the elements of $\mathfrak{M}$ corresponding to the first $j(n)$ columns of the relation matrix. The $\mathfrak{M}_{n}$ form a monotone increasing sequence of $f . g$. submodules of $\mathfrak{M}$ whose union is $\mathfrak{M}$.

A sufficient condition for a relation matrix to be of the above form is the following.

5. Let $\mathfrak{M}$ be a c.g. $R$-module, $\left[a_{i j}\right]$ a relation matrix, $\mathfrak{F}$ a free $R$-module whose basis elements correspond to the columns of $\left[a_{i j}\right]$, and $\Re$ the submodule of $\mathfrak{F}$ whose generators $r_{1}, r_{2}, \ldots$ correspond to the rows of $\left[a_{i j}\right]$. Hence, $\mathfrak{M}=\mathfrak{F} / \mathfrak{R}$. If $i(1) \leqq i(2) \leqq \cdots$ and $j(1) \leqq j(2) \leqq \cdots$ are monotone increasing sequences of positive integers such that:

(a) $a_{1 j}=a_{2 j}=\cdots=a_{i(n), j}=0$, for $j>j(n)$,

(b) the row vectors $r_{i(n)+1}, \ldots, r_{i(n+1)}$ are linearly independent modulo $\mathfrak{F}_{j(n)}$, where $\mathfrak{F}_{j(n)}$ denotes the submodule of $\mathfrak{F}$ generated by the first $j(n)$ columns of $\left[a_{i j}\right]$, then the first $i(n)$ rows and $j(n)$ columns of $\left[a_{i j}\right]$ constitute a relation matrix of a f.g. submodule $\mathfrak{M}_{m}$ of $\mathfrak{M}$ generated by the elements of $\mathfrak{M}$ corresponding to the first $j(n)$ columns of $\left[a_{i j}\right]$.

Propositions II.C.4 and 5 are slight generalizations of those found in [6]. Finally, the local topologies are computed by use of the following.

6. If $\mathfrak{M}$ is a c.g. R-module, A one of its relation matrices, and $\mathfrak{M}_{m}$ the f.g. submodule of $\mathfrak{M}$ generated by the elements of $\mathfrak{M}$ corresponding to the first $n$ columns of $A$, then a relation matrix of $\mathfrak{M} / \mathfrak{M}_{n}$ results if the first $n$ columns of $A$ are omitted.

III. Z-groups modulo local equivalence. In this section all groups are assumed to be countably presentable.

A. Z-groups and local equivalence.

1. Definition. A $Z$-group $[G, \alpha]$ is a group $G$ together with a homomorphism $\alpha$ of $G$ onto the free cyclic group $Z$.

2. Definition. Let $[G, \alpha]$ and $[H, \beta]$ be Z-groups. Then a Z-homomorphism $\phi:[G, \alpha] \rightarrow[H, \beta]$ of $[G, \alpha]$ into $[H, \beta]$ is a homomorphism $\phi: G \rightarrow H$ of $G$ into $H$ such that $\beta \phi=\alpha$.

3. Definition. A normal subgroup $N$ of a group $G$ is finitely implicated if there exists a finite collection of elements of $G$ for which $N$ is the smallest normal subgroup of $G$ containing this collection.

4. Definition. Two $Z$-groups $[G, \alpha]$ and $[H, \beta]$ are locally equivalent if there exists a finite sequence of $Z$-groups $[G, \alpha]=\left[G_{0}, \alpha_{0}\right],\left[G_{1}, \alpha_{1}\right],\left[G_{2}, \alpha_{2}\right], \ldots,\left[G_{n}, \alpha_{n}\right]$ $=[H, \beta]$ such that for every $i, 0<i \leqq n$, there exists either a $Z$-homomorphism with finitely implicated kernel of $\left[G_{i-1}, \alpha_{i-1}\right]$ onto $\left[G_{i}, \alpha_{i}\right]$ or one from $\left[G_{i}, \alpha_{i}\right]$ onto $\left[G_{i-1}, \alpha_{i-1}\right]$.

It readily follows that:

5. Two Z-groups $[G, \alpha]$ and $[H, \beta]$ are locally equivalent if and only if there exist a Z-group $[K, \gamma]$ and Z-homomorphisms with finitely implicated kernels of $[G, \alpha]$ onto $[K, \gamma]$ and of $[H, \beta]$ onto $[K, \gamma]$. 
It is worth noting that finitely generated Z-groups are trivial modulo local equivalence; for:

6. Let $[G, \alpha]$ be a Z-group. If $G$ is finitely generated, then the kernel of $\alpha$ is finitely implicated. Thus, $[G, \alpha]$ is locally equivalent to the trivial Z-group $[Z$, identity].

Proof. Let $\left\{g_{1}, g_{2}, \ldots, g_{n}\right\}$ be a finite set of generators of $G$. Without loss of generality it may be assumed that $\alpha\left(g_{i}\right)=t, i=1,2, \ldots, n$, where $t$ is the generator of $Z$. All that need be shown is that the kernel of $\alpha$ is implicated by $\left\{g_{i} \cdot g_{j}^{-1}\right\}$. Q.E.D.

B. The module of a Z-group. In [6] Brody associated a module with a group as follows.

1. Definition. Let $[G, \alpha]$ be a $Z$-group and $(x: r)$ one of the countable presentations of $G$. Then the matrix $[\partial r / \partial x]^{\alpha \phi}$, where $\partial r / \partial x$ denotes the free derivative of $r$ with respect to $x$ [8], may be considered as a relation matrix of a $J Z$-module, called the module of $[G, \alpha]$ and written $\mathfrak{M}[G, \alpha]$, where $J Z$ is the group ring of $Z$ $([8$, p. 84$])$. It is easily verified $([6$, p. 145$])$ that the generalized Tietze transformations of types (0), (I), and (II) of ( $x: \boldsymbol{r})$ induce elementary transformations of types (0), (I), and (II) respectively on the relation matrix $[\partial \boldsymbol{r} / \partial \boldsymbol{x}]^{\alpha \phi}$ of $[G, \alpha]$. Hence, $\mathfrak{M}[G, \alpha]$ depends only on $[G, \alpha]$ and not on the particular presentation of $G$ chosen.

2. Definition. Let $[G, \alpha]$ and $[H, \beta]$ be Z-groups and $\theta:[G, \alpha] \rightarrow[H, \beta]$ a $Z$-homomorphism. Let $(\boldsymbol{x} ; \boldsymbol{y}: \boldsymbol{r} ; \boldsymbol{s})_{\phi_{0} \phi_{1}}$ be a countable presentation of $\theta([12$, p. 412]). Let $\delta x$ denote the element of $\mathfrak{M}[G, \alpha]$ corresponding to the column $(\partial r / \partial x)^{\alpha \phi_{0}}$ of the relation matrix $(\partial \boldsymbol{r} / \partial \boldsymbol{x})^{\alpha \phi_{0}}$. Similarly, let $\delta x^{*}$ denote the element of $\mathfrak{M}[H, \beta]$ corresponding to the column $[\partial(r \cup s) / \partial x]^{\beta \phi_{1}}$ of $[\partial(r \cup s) / \partial(x \cup y)]^{\beta \phi_{1}}$. It may easily be shown that a well-defined homomorphism $\theta_{*}: \mathfrak{M}[G, \alpha] \rightarrow \mathfrak{M}[H, \beta]$ independent of the presentation of $\theta$ chosen may be defined by $\theta_{*}(\delta x)=\delta x^{*} . \theta_{*}$ is called the homomorphism induced by $\theta$.

The reader may readily supply the proof for the following.

3. If $\theta$ is onto, then so is $\theta_{*}$. If the kernel of $\theta$ is finitely implicated, then the kernel of $\theta_{*}$ is finitely generated.

4. Definition. Two modules $\mathfrak{M}$ and $\mathfrak{R}$ are locally equivalent if there exist finitely generated submodules $\mathfrak{M}^{*}$ and $\mathfrak{N}^{*}$ of $\mathfrak{M}$ and $\mathfrak{N}$ respectively such that $\mathfrak{M} / \mathfrak{M}^{*}$ and $\mathfrak{N} / \mathfrak{R}^{*}$ are isomorphic.

The above is found to be an equivalence relation.

The significance of the above definition can be seen from the following, a consequence of III.B.3.

7. If two Z-groups are locally equivalent, then their corresponding modules are locally equivalent.

C. Modules modulo local equivalence.

1. Definition. An elementary transformation of type (IV) adjoins finitely many new rows to the relation matrix, each new row containing only finitely many nonzero entries. 
2. Two c.g. modules are locally equivalent if and only if any relation matrix of either module may be obtained from any relation matrix of the other by the application of a finite sequence of elementary transformations of types (0), (I), (II), and (IV).

Proof. A relation matrix of a quotient module $\mathfrak{M} / \mathfrak{M}^{*}$, where $\mathfrak{M}^{*}$ is $\mathrm{f} . \mathrm{g}$., can be obtained by adjoining finitely many rows to a relation matrix of $\mathfrak{M}$. Q.E.D.

Next we seek invariants of modules modulo local equivalence.

3. If two c.g. modules are locally equivalent, then their $k$ th divisor chains are equivalent, for every $k \geqq 0$.

Proof. Let $\mathfrak{M}$ be a c.g. module and $\mathfrak{M}^{*}$ a f.g. submodule. All that need be demonstrated is that $\mathfrak{M}$ and $\mathfrak{M} / \mathfrak{M}^{*}$ have equivalent $k$ th divisor chains. We leave this to the reader. Q.E.D.

It now follows from I.D.3 that the local topologies are invariants of modules modulo local equivalence.

IV. Algebraic invariants of local type. Algebraic invariants of the local types of arcs at singular interior points are now constructed. Throughout this section $k$ and $k^{\prime}$ will denote oriented arcs and $p$ and $p^{\prime}$ singular interior points of $k$ and $k^{\prime}$ respectively.

A. Local Z-groups.

1. There exist arbitrarily small open neighborhoods $U$ of $p$ which satisfy the following conditions with respect to $k$ :

(a) the boundary $\partial U$ of $U$ is a tame 2-sphere,

(b) $k \cap \partial U$ consists of finitely many points,

(c) $k$ pierces [19] $\partial U$ at each point of $k \cap \partial U$,

(d) the end points of $k$ do not lie in the closure $\bar{U}$ of $U$.

Such neighborhoods will be termed acceptable neighborhoods of $p$ with respect to $k$.

Proof. From ([14, Theorem 8]) it follows that there exists a locally finite triangulation $T$ of $S^{3}-p$ for which $k-p$ is a subcomplex. Let $N$ be an arbitrarily small closed cube with center at $p$, not containing the end points of $k$. Then by ([3, p. 56]) there exists for every $\varepsilon>0$ a homeomorphism $f_{\varepsilon}^{*}$ of $S^{3}-p$ onto itself moving no point by as much as $\varepsilon$ which maps the boundary of $N$ onto a polyhedron of $S^{3}-p$ under the triangulation $T$. Extending $f_{\varepsilon}^{*}$ to a homeomorphism $f_{\varepsilon}$ of $S^{3}$ upon itself, it follows that the interior $U$ of $f_{\varepsilon}(N)$ is an open neighborhood of $p$ which satisfies condition (a). By choosing $\varepsilon$ sufficiently small it may be assumed that $U$ also satisfies (d). Finally by applying sufficiently small deformations to $\bar{U}$, $U$ can be made to satisfy (b) and (c). Q.E.D.

2. Definition. Let $U$ be an acceptable neighborhood of $p$ with respect to $k$. Because of (b) and (d) of IV.A.1, $k \cap \bar{U}$ may be considered as a singular relative 1-cycle of $\bar{U}$ modulo $\partial \bar{U}$. By Alexander's theorem ([15, p. 25]) condition (a) implies that $\bar{U}$ is a closed 3-cell. Hence, the linking number of the relative 1-cycle $k \cap \bar{U}$ with any 1-cycle of $U-k$ may be defined [18]. This linking number may then be used to define a homomorphism of the first homology group $H_{1}(U-k)$ of 
$U-k$ onto $Z$ ([21, p. 280]). The composition of this homomorphism with the natural homomorphism of the fundamental group $\Pi(U-k)$ of $U-k$ onto $H_{1}(U-k)$ will be called the homomorphism determined by $k$.

3. Definition. Let $U$ be an acceptable neighborhood of $p$ with respect to $k$. A local Z-group of $k$ at $p$ is the group $\Pi(U-k)$ together with the homomorphism determined by $k$.

REMARK. IV.A.1 guarantees the existence of at least one local Z-group.

B. The fundamental invariance theorem. In this section it will be demonstrated that a local $Z$-group modulo local equivalence is an invariant of local type.

1. Let $U$ and $V$ be two acceptable neighborhoods of $p$ with respect to $k$ such that the closure $\bar{V}$ of $V$ is in $U$. Then $\Pi(U-\bar{V}-k)$ is finitely generated.

Proof. (By Alexander duality and ([16, p. 48]) $U-\bar{V}-k$ is connected.) $\bar{U}-V$ is obviously a compact 3-manifold. It follows from ([19, Theorem 1]) that $k \cap(\bar{U}-V)$ is a locally tame closed subset of $\bar{U}-V$. Thus, by $([3$, p. 154]) $\bar{U}-V$ has a locally finite (hence, finite) triangulation under which $k \cap(\bar{U}-V)$ is a polyhedron. Finally, from $([20$, p. 13]) it may be concluded that $\Pi(\bar{U}-V-k)$ and hence $\Pi(U-\bar{V}-k)$ are finitely generated. Q.E.D.

Since the intersection number of a 1-chain with a 2-chain is merely the number of algebraic penetrations, it follows that:

2. Let $U$ and $V$ be two acceptable neighborhoods of $p$ with respect to $k$ such that $V \subset U$. Then $\nu^{\prime} i_{*}=\nu$, where $i_{*}: \Pi(V-k) \rightarrow \Pi(U-k)$ is the homomorphism induced by inclusion and $\nu: \Pi(V-k) \rightarrow Z$ and $\nu^{\prime}: \Pi(U-k) \rightarrow Z$ are the homomorphisms determined by $k$.

3. Fundamental invariance theorem. If $k$ at $p$ is of the same local type as $k^{\prime}$ at $p^{\prime}$, then each local Z-group of $k$ at $p$ is locally equivalent to each local Z-group of $k^{\prime}$ at $p^{\prime}$.

The proof is in two parts.

PART ONE. Any two local Z-groups of $k$ at $p$ are locally equivalent.

Proof. Let $U$ and $V$ be acceptable neighborhoods of $p$ with respect to $k$ such that the closure $\bar{V}$ of $V$ is in $U$. Then the proof of the statement of part one reduces to demonstrating that the local $Z$-groups corresponding to $U$ and $V$ are locally equivalent.

By IV.A.1 an acceptable neighborhood $W$ of $p$ with respect to $k$ may be selected so that its closure $\bar{W}$ lies in $V$. Consider the diagram:

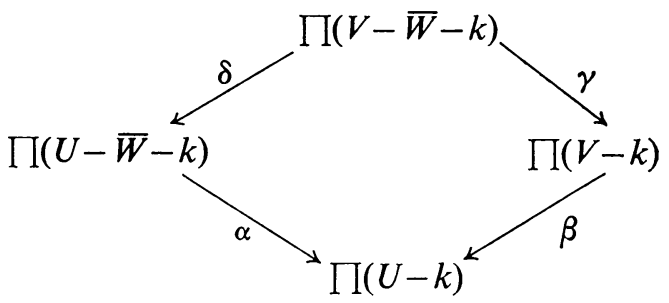

where $\alpha, \beta, \gamma$, and $\delta$ are the homomorphisms induced by inclusions. By the Van 
Kampen theorem [7] $\Pi(U-k)$ is the direct limit of the system consisting of the three upper groups and their connecting homomorphisms. Furthermore, the homomorphisms $\alpha$ and $\beta$ are the uniquely determined homomorphisms of the system onto the direct limit.

Let $\mu: \Pi(V-k) \rightarrow Z$ and $\zeta: \Pi(U-k) \rightarrow Z$ be the homomorphisms determined by $k$. Let $\nu: \Pi(V-\bar{W}-k) \rightarrow Z$ and $\varepsilon: \Pi(U-\bar{W}-k) \rightarrow Z$ be the compositions $\mu \gamma$ and $\zeta \alpha$, respectively. Then from IV.B.2 it follows that $\zeta \beta=\mu$ and hence $\varepsilon \delta=\nu$.

Now let $Q$ be the consequence ([8, p. 38]) of $\gamma \operatorname{Ker} \nu$ and let $\omega$ be the natural homomorphism of $\Pi(V-k)$ onto $\Pi(V-k) / Q$. Since $\gamma \operatorname{Ker} \nu \subset \operatorname{Ker} \omega$, there exists a homomorphism $\sigma: Z \rightarrow \prod(V-k)$ so that $\sigma \nu=\omega \gamma$. Moreover, since $\operatorname{Ker} \omega \subset \operatorname{Ker} \mu$, there exists a homomorphism $\rho: \Pi(V-k) / Q \rightarrow Z$ of $\Pi(V-k) / Q$ onto $Z$ so that $\rho \omega=\mu$. It should be noted that $\rho \sigma=1$. For $\rho \sigma \nu=\rho \omega \gamma=\mu \gamma=\nu$.

Collecting these observations together, we have the diagram of Figure 1, where

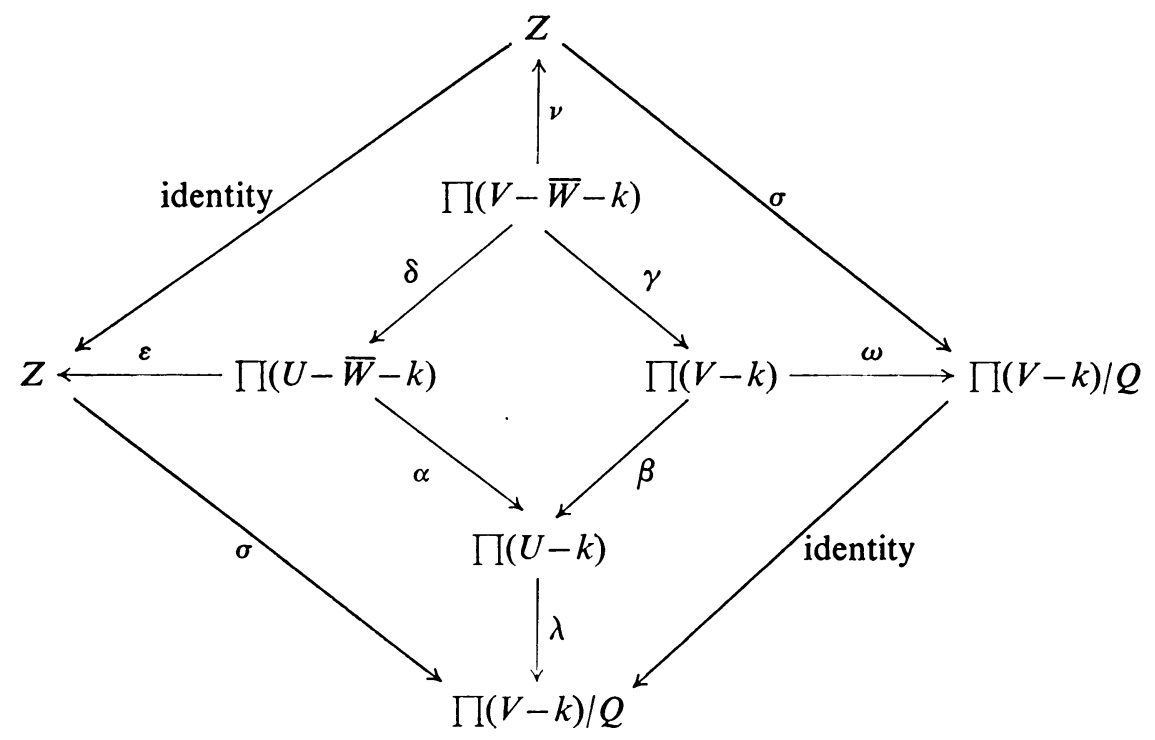

FIGURE 1

it is now observed that $\Pi(V-k) / Q$ is the direct limit of the system consisting of the three outer groups and their connecting homomorphisms, where $\sigma$ and the identity are the uniquely determined homomorphisms of the system onto the direct limit $\Pi(V-k) / Q$, and where $\lambda$ is defined as the uniquely determined homomorphism of the direct limit $\Pi(U-k)$ into $\Pi(V-k) / Q$. That $\lambda$ is actually onto may easily be verified.

The kernel of $\lambda$ will now be shown to be the consequence $Q^{*}$ of $[(\alpha \operatorname{Ker} \varepsilon)$ $\cup(\beta \operatorname{Ker} \omega)]$. Let $\lambda^{*}: \Pi(U-k) \rightarrow \prod(U-k) / Q^{*}$ be the natural homomorphism. Since $\operatorname{Ker} \lambda^{*} \subset \operatorname{Ker} \lambda$, there exists a homomorphism $\chi: \Pi(U-k) / Q^{*} \rightarrow \Pi(V-k) / Q$ (which is an epimorphism) such that $\chi^{*}=\lambda$. Since $\alpha \operatorname{Ker} \varepsilon \subset \operatorname{Ker} \lambda^{*}$ and $\beta \operatorname{Ker} \omega$ 
$\subset \operatorname{Ker} \lambda^{*}$, there exist homomorphisms $\phi: Z \rightarrow \Pi(U-k) / Q^{*}$ and $\psi: \Pi(V-k) / Q$ $\rightarrow \prod(U-k) / Q^{*}$ such that $\phi \varepsilon=\lambda^{*} \alpha$ and $\psi \omega=\lambda^{*} \beta$. By means of $\phi, \psi, \chi$ any homomorphism of the outer system into a group may be factored through $\Pi(U-k) / Q^{*}$. Hence, $\Pi(U-k) / Q^{*}$ is the direct limit of this system. Thus $\operatorname{Ker} \lambda=Q^{*}=$ consequence of $[(\alpha \operatorname{Ker} \varepsilon) \cup(\beta \operatorname{Ker} \omega)]$.

That the Z-groups corresponding to $U$ and $V$ are locally equivalent can be established as follows. Since $\rho \lambda \alpha=\rho \sigma \varepsilon=\varepsilon=\zeta \alpha$ and $\rho \lambda \beta=\rho \omega=\mu=\zeta \beta$ and $\operatorname{Im}(\alpha)$ $\cup \operatorname{Im}(\beta)$ generates $\Pi(U-k), \rho \lambda=\zeta$. From above $\rho \omega=\mu$. Hence, $\lambda$ and $\omega$ are both $Z$ epimorphisms. By IV.B.1, $\Pi(V-\bar{W}-k)$ and $\Pi(U-\bar{W}-k)$ are finitely generated. Thus, according to III.A.6, ker $\nu$ and $\operatorname{ker} \varepsilon$ are finitely implicated. Hence, Ker $\omega$ (which is the consequence of $\gamma \operatorname{Ker} \nu$ ) and $\operatorname{ker} \lambda$ (which is the consequence of $[(\alpha \operatorname{ker} \varepsilon) \cup(\beta \operatorname{Ker} \omega)])$ are finitely implicated. The conclusion now follows from III.A.5. This completes the proof of part one.

PART Two. There is a local $Z$-group of $k$ at $p$ which is locally equivalent to a local Z-group of $k^{\prime}$ at $p^{\prime}$.

Proof. By hypothesis there exist open neighborhoods $U$ and $U^{\prime}$ of $p$ and $p^{\prime}$ respectively and an orientation preserving homeomorphism $\phi$ of $U$ onto $U^{\prime}$ which maps $U \cap k$ onto $U^{\prime} \cap k^{\prime}$ and $p$ upon $p^{\prime}$. By IV.A.1 there exists an acceptable neighborhood $V$ of $p$ with respect to $k$ such that its closure $\bar{V}$ lies in $U$. Hence, $V^{\prime}=\phi(V)$ is an open neighborhood of $p$ which obviously satisfies conditions (b), (c), and (d) of IV.A.1. Since the boundary of $V^{\prime}$ is locally tame, it follows from [4] that $V^{\prime}$ satisfies condition (a) of IV.A. 1 and hence, $V^{\prime}$ is an acceptable neighborhood of $p^{\prime}$ with respect to $k^{\prime}$.

Now $\phi$ induces an isomorphism $\phi_{*}$ of $\Pi(V-k)$ onto $\Pi\left(V^{\prime}-k^{\prime}\right)$. Since $\phi$ is orientation preserving, it preserves the linking number. Hence, $\nu^{\prime} \phi_{*}=\nu$, where $\nu, \nu^{\prime}$ are the homomorphisms determined by $k$ and $k^{\prime}$, respectively. Thus, $\phi_{*}$ is a $Z$-isomorphism.

C. Further invariants of local type. At this point we would like to employ the results of §III. These results however are only applicable to countably generated $Z$-groups. Fortunately, every local Z-group is countably generated. This follows readily by applying IV.A.1, IV.B.1, and the Van Kampen theorem [7] and by observing that the direct limit of a countable system of finitely generated groups is countably generated.

1. Definition. A local module of $k$ at $p$ is the module of a local Z-group of $k$ at $p$.

From III.B.7 and the fundamental invariance theorem IV.B.3 it follows that a local module modulo local equivalence is an invariant of local type.

2. Definition. For every integer $n \geqq 0$, an $n$th divisor chain of $k$ at $p$ is an $n$th divisor chain of a local module of $k$ at $p$.

We may conclude from III.C. 3 that each $n$th divisor chain modulo equivalence is an invariant of local type.

Since local topologies are invariants of modules modulo local equivalence, we have yet another invariant of local type, namely: 
3. Definition. For every integer $n \geqq 0$, the $n$th local topology of $k$ at $p$, written $\Lambda_{n}(k, p)$, is defined as the $n$th local topology of any module of $k$ at $p$.

V. Applications. It is now possible to consider some of the applications of the algebraic invariants developed in the previous section.

A. Wilder arcs.

1. Definition. Let $k$ be an arc and $p$ a point of $k$. The penetration index [1], [2] of $k$ at $p$, written $P(k, p)$, is the smallest cardinal number $n$ such that there are arbitrarily small 2-spheres enclosing $p$ and containing no more than $n$ points of $k$.

2. Definition. Let $p$ be a singular interior point of an $\operatorname{arc} k$. Then $k$ is Wilder arc if $P(k, p)=2$.

3. A method of decomposing an arbitrary Wilder arc $k$ with singular interior point p into a sequence $K_{n}$ of tame knots is now given.

Since $P(k, p)=2$ there is a sequence of arbitrarily small 2-spheres $S_{n}$, each $S_{n}$ enclosing $p$ and containing exactly two points $a_{n}$ and $b_{n}$ of $k$ such that the oriented subarc from $a_{n}$ to $b_{n}$ has the induced orientation of $k$. By ([1, p. 34]) it may be assumed that each $S_{n}$ is tame and pierced at $a_{n}$ and $b_{n}$ by $k$. Let $U_{n}$ denote the complementary domain of $S_{n}$ containing $p$. (Hence, by Alexander's theorem ([15, p. 25]) $U_{n}$ is an open 3-cell.) Thus, the $U_{n}$ 's form a sequence of acceptable neighborhoods of $p$ with respect to $k$ such that:

(a) the closure $\bar{U}_{n}$ of $U_{n}$ lies in $U_{n-1}$,

(b) $\cap U_{n}=p$,

(c) $k$ cuts the boundary of $U_{n}$ in just two points $a_{n}$ and $b_{n}$, and

(d) by [19] $k \cup S_{n}$ is locally tame at $a_{n}$ and $b_{n}$.

$k$ is decomposed as follows. $\bar{U}_{n-1}-U_{n}$ intersects $k$ in two oriented arcs $A_{n}$ from $a_{n-1}$ to $a_{n}$ and $B_{n}$ from $b_{n}$ to $b_{n-1}$. On $S_{n}$ select an arc $J_{n}$ from $a_{n}$ to $b_{n}$. By (d) the oriented simple closed curve $K_{n}=A_{n} \cup J_{n} \cup B_{n} \cup J_{n-1}^{-1}$ is a tame knot. The sequence of tame knots $K_{n}$ is the decomposition of $k$ sought.

4. Let $k$ be $a$ Wilder arc and $p$ its singular interior point. Let $K_{n}$ be a decomposition of $k$ into a sequence of tame knots as accomplished above. Then each local module of $k$ at $p$ is locally equivalent to a direct sum $\bigoplus_{n=1}^{\infty} \mathfrak{M}_{n}$ of JZ-modules $\mathfrak{M}_{n}$, where $J Z \oplus \mathfrak{M}_{n}$ is the Alexander module of $K_{n}$.

Proof. Let $U_{n}$ be the sequence of open 3-cells constructed in V.A.3. By repeated application of the Van Kampen theorem [7] it may be shown that $\Pi\left(U_{0}-k\right)$ is the infinite amalgamated free product:

$$
\Pi\left(U_{0}-k\right) \simeq \Pi\left(U_{0}-\bar{U}_{1}-k\right) *_{z} \Pi\left(U_{1}-\bar{U}_{2}-k\right) *_{z} \cdots,
$$

where it should be observed that the groups $\Pi\left(U_{n-1}-\bar{U}-k\right)$ are respectively the knot groups of the knots $K_{n}$.

Now select a Wirtinger presentation $\left(a_{n}, x_{n}: r_{n}\right)$ of $\Pi\left(U_{n-1}-\bar{U}_{n}-k\right)$ such that for all $n$ each generator is mapped by the homomorphism determined by $k$ onto 
the same generator of $Z$ and such that all $a_{n}$ are images of the same generator of the amalgamating group $Z$. Thus, a presentation of $\Pi\left(U_{0}-k\right)$ is:

$$
\left(a_{1}, \boldsymbol{x}_{1}, a_{2}, \boldsymbol{x}_{2}, \ldots: \boldsymbol{r}_{1}, a_{1} a_{2}^{-1}, \boldsymbol{r}_{2}, a_{2} a_{3}^{-1}, \ldots\right) \text {. }
$$

Applying generalized Tietze transformations, we have an equivalent presentation:

$$
\left(a, x_{1}, x_{2}, \ldots: r_{1}^{*}, r_{2}^{*}, \ldots\right) \text {, }
$$

where $\boldsymbol{r}_{n}^{*}$ denotes $\boldsymbol{r}_{n}$ with $a_{n}$ replaced by $a$. The corresponding relation matrix for the module associated with $\Pi\left(U_{0}-k\right)$ is:

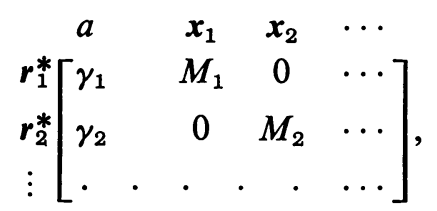

where $\left[\gamma_{n} M_{n}\right]$ is an Alexander matrix of $K_{n}$, i.e., a relation matrix of the Alexander module of the knot group $\Pi\left(U_{n-1}-\bar{U}_{n}-k\right)$. It follows from the fundamental formula of free calculus ([10, p. 551]) that the sum of the columns of $M_{n}$ is $-\gamma_{n}$. Hence performing a matrix transformation of type III, we may replace the first column of the above matrix by a column of zeros. Thus, the module associated with $\Pi\left(U_{0}-k\right)$ is the infinite direct sum $J Z \oplus \mathfrak{M}_{1} \oplus \mathfrak{M}_{2} \oplus \mathfrak{M}_{3} \oplus \cdots$, where $\mathfrak{M}_{n}$ is the module corresponding to the matrix $M_{n}$. Observing that $\left[0 M_{n}\right]$ is an Alexander matrix of $K_{n}$-hence, that $J Z \oplus \mathfrak{M}_{n}$ is the Alexander module of $K_{n}$, we have the conclusion. Q.E.D.

By II.B.3 and 7,

5. $\prod_{n=1}^{\infty} \nabla_{1}\left(K_{n}\right) \succ \prod_{n=2}^{\infty} \nabla_{1}\left(K_{n}\right) \succ \prod_{n=3}^{\infty} \nabla_{1}\left(K_{n}\right) \succ \cdots$ is a 0 th divisor chain of $k$ at $p$, where $\nabla_{1}\left(K_{n}\right)$ is the 1 st divisor topology of the tame knot $K_{n}$. Hence, the 0th divisor chains of $k$ at $p$ are comparable; and thus, $a$ th comparing topology of $k$ at $p$ exists. One such comparing topology is $\prod_{n=1}^{\infty} \nabla_{1}\left(K_{n}\right)$.

Hence, the 0th comparing topology modulo similarity is for Wilder arcs yet another invariant of local type.

V.A.5 together with I.D.5 give the following algebraic analogue to the geometric classification of Wilder arcs of Fox and Harrold [14].

6. Let $k$ and $k^{\prime}$ be Wilder arcs and $\sigma$ and $\sigma^{\prime}$ comparing topologies of each respectively. If $k$ and $k^{\prime}$ are of the same global type, then

(a) $\sigma$ and $\sigma^{\prime}$ have the same set of prime factors with infinite exponent, and

(b) $e(p, \sigma)=e\left(p, \sigma^{\prime}\right)$ for almost all (up to associates) irreducible elements of JZ.

7. An irreducible element of $\mathrm{JZ}$ is a prime factor of the 0th local topology of $k$ at $p$ if and only if it divides infinitely many of the Alexander polynomials of the knots $K_{n}$. Moreover, each such prime factor has infinite exponent.

B. Mixed-composites of arcs. A method of combining arcs is now defined and investigated. Throughout the remainder of this paper every cone in $S^{3}$ to be considered will be assumed to be locally tame at all of its points except possibly its vertex. 
1. Definition. By the standard half-cone $C_{R}^{\#}$ will be meant the set of points $\left\{(x, y, z): y^{2}+z^{2} \leqq x^{2}, 0 \leqq x \leqq 1\right\}$.

2. Let $C$ be a half-cone. An axis of $C$ is an image of $\{(x, 0,0): 0 \leqq x \leqq 1\}$ under a homeomorphism of $C_{R}^{\#}$ onto $C$ which maps base upon base and vertex upon vertex.

It is easily verified that the local type of the axis of a cone at the cone's vertex is independent of the homeomorphism chosen.

3. Definition. Let $p$ be a singular interior point of an arc $k$. A half-cone containing $k$ will be said to enclose $k$ at $p$ if the only points of $k$ lying on the cone's boundary are $p$ which is the cone's vertex and the end points of $k$ which lie on the interior of the cone's base. (See $k_{0}$ in Figure 2.)

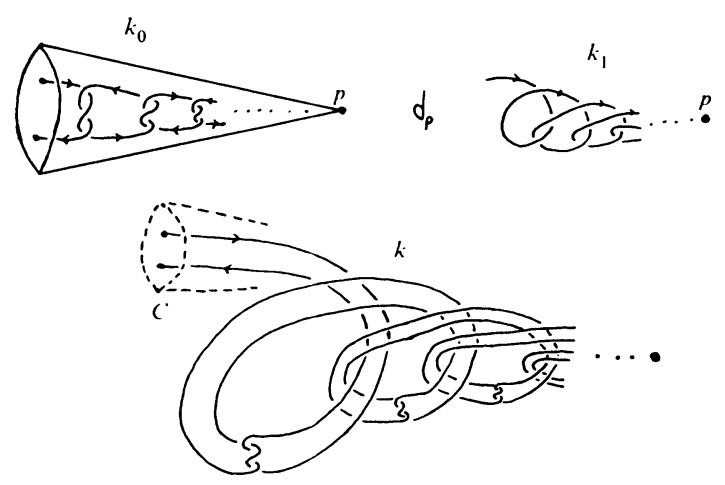

Figure 2. A Mixed Composite

4. Let $p$ be a singular interior point of arcs $k$ and $k_{0}$ and a singular end point of an arc $k_{1} . k$ at $p$ is a mixed-composite of $k_{0}$ with respect to $k_{1}$ at $p$, written $k_{0} \quad{ }_{p} k_{1}$, if there exists a half-cone $C$ enclosing $k$ at $p$ such that:

(a) there is an orientation preserving homeomorphism $f$ of $C$ onto $C_{R}^{\#}$ mapping base onto base and vertex onto vertex such that $f(k)$ at the origin is of the same local type as $k_{0}$ at $p$, and

(b) an axis of $C$ is of the same local type at $p$ as $k_{1}$ at $p$. (See Figure 2.)

REMARK. Let $f$ and $g$ be any two homeomorphisms of $C$ onto $C_{R}^{\# \text { mapping }}$ base onto base and vertex onto vertex. Since the homeomorphism $g f^{-1}$ of $C_{R}^{\#}$ onto itself can be extended in the usual fashion to a homeomorphism of $S^{3}$ upon itself, it follow's that the above definition is independent of the homeomorphism $f$ selected.

5. Let $p$ be a singular interior point of arcs $k$ and $k_{0}$ and a singular end point of $k_{1}$. If $k$ at $p$ is a mixed-composite of $k_{0}$ with respect to $k_{1}$ at $p$, then $k$ and $k_{0}$ both have the same local Z-groups at $p$.

V.B.5 is an immediate consequence of:

6. Let $C$ be a half-cone which encloses $k$ at $p$. Then $\Pi(C-k)$ as a Z-group is locally equivalent to each local Z-group of $k$ at $p$. 
Proof. Construct a half-cone $C^{*}$ containing $C$ such that (a) the only points of $C$ lying on the boundary of $C^{*}$ are the points of $C^{\prime}$ 's base, all of which lie on the base of $C^{*}$, and the vertex of $C$ which coincides with the vertex of $C^{*}$, and (b) $C$ is a deformation retract of $C^{*}$. Also construct an acceptable neighborhood $U$ of $p$ with respect to $k$ such that the base of $C^{*}$ and no other points of $C^{*}$ lie on the boundary of $U$. This construction is possible since $C$ is locally tame everywhere except possibly its vertex.

It is immediate that $\Pi(U-C)$ is trivial, that $\Pi\left[(U-C) \cap\left(\left(\operatorname{Int} C^{*}\right)-k\right)\right]$ is $Z$ (where Int $C^{*}$ denotes the interior of $\left.C^{*}\right)$, and that $\Pi\left[\left(\operatorname{Int} C^{*}\right)-k\right]$ and $\Pi(C-k)$ are $Z$-isomorphic. Thus, by the Van Kampen theorem [7], $\Pi(U-k)$ is the direct limit of:

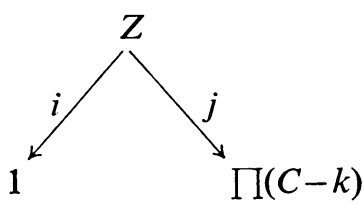

where $i$ and $j$ are the homomorphisms induced by inclusions. It now follows that $\Pi(C-k)$ and $\Pi(U-k)$ are locally equivalent $Z$-groups. Q.E.D.

The following theorem indicates that there is very little relation between the algebraic invariants of this paper and the penetration index.

7. TheOREM. If $k$ at $p$ is a mixed-composite of $k_{0}$ with respect to $k_{1}$ at $p$, then $P(k, p) \geqq P\left(k_{0}, p\right) P\left(k_{1}, p\right)$.

C. Interior composites of arcs. The following composite of arcs is analogous to the companions of tame knots ([22, p. 192]).

1. Definition. By the standard cone $C^{\#}$ will be meant $\left\{(x, y, z): y^{2}+z^{2} \leqq x^{2} \leqq 1\right\}$.

2. Definition. Let $C$ be a cone. An axis $k$ of $C$ is the image of $\{(x, 0,0):|x| \leqq 1\}$ under a homeomorphism of $C^{\#}$ onto $C$ which maps lateral surfaces onto corresponding lateral surfaces.

It is a straightforward exercise to verify that the axis of a cone is uniquely determined up to local type.

3. Definition. Let $p$ be an interior point of an arc $k$. A point $q$ of $k$ (distinct from $p$ ) will be said to lie to the left or to the right of $p$ accordingly as the oriented subarc from $q$ to $p$ has the same or the opposite orientation of that induced by $k$. By convention $p$ lies both to the left and to the right of itself. The arc consisting of those points to the left of $p$ and that consisting of those to the right of $p$ will be called respectively the left half, written $k_{L}$, and the right half, written $k_{R}$, of $k$ with respect to $p$.

4. Definition. A cone containing $k$ will be said to enclose $k$ at $p$ if the only points of $k$ lying on the cone's boundary are $p$ which coincides with the cone's vertex and the end points of $k$ which lie on the respective bases of $C$. The half-cones 
of $C$ which contain the left and right halves of $k$ are denoted by $C_{L}$ and $C_{R}$, respectively, (see Figure 3).

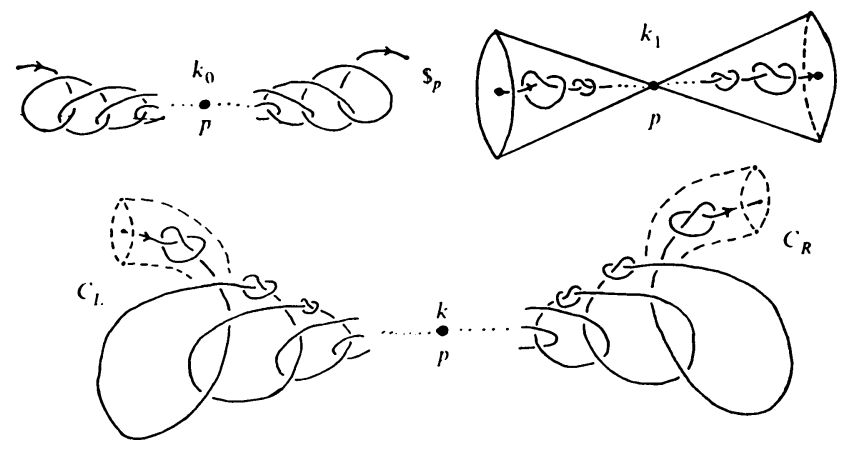

Figure 3. An Interior Composite

5. Definition. Let $p$ be a singular interior point of an arc $k$ such that $k$ pierces a tame disc at $p$. From this it follows that there exists a tame cone $C=C_{L} \cup C_{R}$ enclosing $k$ at $p$. Remove the right (or left) half of $k$ with respect to $p$ and adjoin at $p$ an axis of $C_{R}$ (or $C_{L}$ ). The resulting arc $k^{L}$ (or $k^{R}$ ) is called a left (or a right) factor of $k$ at $p$ (although it is not unique).

6. Definition. Let $p$ be a singular interior point of an arc $k$. $k$ is left (or right) tame at $p$ if there exists a tame cone $C$ which encloses the left (or right) half of $k$ at $p$ so that the left (or right) half of $k$ is tame in $C$.

Remark. Obviously, a left or right tame arc pierces a tame disc.

7. Definition. Let $p$ be a singular interior point of arcs $k, k_{0}$, and $k_{1}$. Then $k$ at $p$ is an interior composite of $k_{0}$ and $k_{1}$ at $p$, written $k=k_{0} \$_{p} k_{1}$, if there exists a cone $C$ enclosing $k$ at $p$ such that:

(a) an axis of $C$ is of the same local type at $p$ as $k_{0}$ at $p$, and

(b) there exists an orientation preserving homeomorphism $f$ of $C$ onto $C^{\#}$ mapping lateral surfaces onto corresponding lateral surfaces such that the image of $k$ under this homeomorphism is of the same local type at the origin as $k_{1}$ at $p$. (See Figure 3.)

REMARK. This definition can easily be seen to be independent of the homeomorphism $f$ chosen.

REMARK. The composition " $\$_{p}$ " of two arcs is not a uniquely defined operation, not even modulo local type.

The following easily verifiable "associative law" indicates that it is sensible to speak of multiple interior composites, the definition of which is left to the reader.

8. Let $p$ be a singular interior point of arcs $k, k_{0}, k_{1}$, and $k_{2}$. Then $k=\left(k_{0} \$_{p} k_{1}\right) \$_{p} k_{2}$ if and only if $k=k_{0} \$_{p}\left(k_{1} \$_{p} k_{2}\right)$.

It is immediate that:

9. Let $p$ be a singular interior point of an arc $k$ such that $k$ pierces $a$ tame disc at $p$. Then $k=k^{R} \$_{p} k^{L}$ and $k=k^{L} \$_{p} k^{R}$. 
10. Let $p$ be a singular interior point of arcs $k, k_{0}$, and $k_{1}$. If $k=k_{0} \$_{p} k_{1}$, then the free product of any local Z-group of $k_{0}$ at $p$ and any local Z-group of $k_{1}$ at $p$ is locally equivalent to each local Z-group of $k$ at $p$.

Proof. Because of V.C.8 and V.C.9, $k_{1}$ may be assumed to be either right or left tame. Let us assume that $k_{1}$ is left tame at $p$. (The proof of the other case is essentially the same.)

Let $C=C_{R} \cup C_{L}$ be the cone corresponding to considering $k$ at $p$ as an interior composite of $k_{0}$ and $k_{1}$ at $p$. Construct a cone $C_{R}^{*} \cup C_{L}^{*}$ containing $C_{R} \cup C_{L}$ such that (a) the only points that the boundary of $C_{R} \cup C_{L}$ has in common with the boundary of $C_{R}^{*} \cup C_{L}^{*}$ are the points of its bases which bases lie on the interior of the corresponding bases of $C_{R}^{*} \cup C_{L}^{*}$ and its vertex which coincides with the vertex of $C_{R}^{*} \cup C_{L}^{*}$, and (b) $C_{R} \cup C_{L}$ is a deformation retract of $C_{R}^{*} \cup C_{L}^{*}$. Also construct an acceptable neighborhood $U$ of $p$ with respect to $k$ such that the bases of the cone $C_{R}^{*} \cup C_{L}$ and no other points of $C_{R}^{*} \cup C_{L}$ lie on the boundary of $U$. Such constructions are possible since $C_{R} \cup C_{L}$ is locally tame everywhere except possibly its vertex.

It is immediate that $\prod\left[\left(U-C_{R}-k_{L}\right) \cap\left(\left(\operatorname{Int} C_{R}^{*}\right)-k_{R}\right)\right]$ is $Z$ (where Int $C_{R}^{*}$ denotes the interior of $\left.C_{R}^{*}\right)$, that $\Pi\left(U-C_{R}-k_{L}\right)$ and $\Pi\left(U-k_{0}\right)$ are $Z$-isomorphic, and that $\Pi\left[\left(\operatorname{Int} C_{R}^{*}\right)-k_{R}\right]$ is $Z$-isomorphic to $\Pi\left(C_{R}-k_{R}\right)$. Thus, by the Van Kampen theorem [7] $\Pi(U-k)$ is the direct limit of the system:

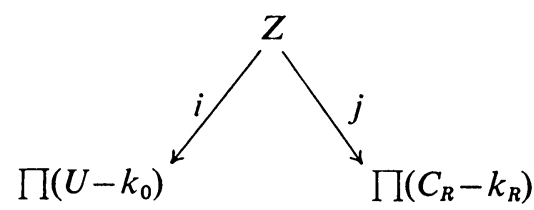

where $i$ and $j$ are the homomorphisms induced by inclusions. From this it readily follows that $\Pi(U-k)$ is locally equivalent to the free product of $\Pi\left(U-k_{0}\right)$ and $\prod\left(C_{R}-k_{R}\right)$.

It will now be shown that $\Pi\left(C_{R}-k_{R}\right)$ is a $Z$-group of $k_{1}$ at $p$. Let $f: C_{R} \cup C_{L} \rightarrow C^{\#}$ be a homeomorphism corresponding to considering $k$ at $p$ as a composite of $k_{0}$ and $k_{1}$. Since $k_{1}$ is left tame, $f$ may be modified so that $f\left(k_{L}\right)=\{(x, 0,0):-1 \leqq x \leqq 0\}$. Let $U^{\prime}$ be an acceptable neighborhood of the origin with respect to $f(k)$ such that all points of the bases of $C^{\#}$ and no other points of $C^{\#}$ lie on the boundary of $U^{\prime}$. Since $C_{R}^{\#}-f\left(k_{R}\right)$ is a deformation retract of $U^{\prime}-f(k), \Pi\left(C_{R}-k_{R}\right)$ and $\Pi\left(U^{\prime}-f(k)\right)$ are $Z$-isomorphic. And since $f(k)$ at the origin is of the same local type as $k_{1}$ at $p$, $\Pi\left(C_{R}-k_{R}\right)$ is a local Z-group of $k_{1}$ at $p$. Q.E.D.

11. The direct sum of any local module of $k_{0}$ at $p$ and any local module of $k_{1}$ at $p$ is locally equivalent to each local module of $k$ at $p$. Hence

$$
\Lambda_{0}(k, p)=\Lambda_{0}\left(k_{0}, p\right) \cdot \Lambda_{0}\left(k_{1}, p\right) .
$$

II.D.5 may be used to compute higher order local topologies of interior composites. 
At this point it is appropriate to explore briefly the penetration index of interior composites.

12. THEOREM. Let $p$ be a singular interior point of an arc $k$. If $k$ pierces a tame disc at $p$, then $P(k, p) \geqq P\left(k_{L}, p\right)+P\left(k_{R}, p\right)$.

13. THEOREM. Let $p$ be a singular interior point of arcs $k, k_{0}$, and $k_{1}$. If $k=k_{0} \$_{p} k_{1}$, then

$$
P(k, p) \geqq P\left(k_{0 L}, p\right) P\left(k_{1 L}, p\right)+P\left(k_{0 R}, p\right) P\left(k_{1 R}, p\right) .
$$

D. Examples. The following method of describing examples is employed. Divide $C^{\#}$ into an infinite number of sections by the family of parallel planes $x= \pm 2^{-n}, n=0,1,2, \ldots$ For each nonnegative integer $n$, let $D_{n}^{+}$and $D_{n}^{-}$denote the sections $2^{-n-1} \leqq x \leqq 2^{-n}$ and $-2^{-n} \leqq x \leqq-2^{-n-1}$, respectively. For convenience let $D^{ \pm}=D_{0}^{ \pm}$. For each nonnegative integer $n$, define orientation preserving homeomorphisms $h_{n}^{+}: D^{+} \rightarrow D_{n}^{+}$and $h_{n}^{-}: D^{-} \rightarrow D_{n}^{-}$as

$$
h_{n}^{ \pm}(x, y, z)=\left(x / 2^{n}, y / 2^{n}, z / 2^{n}\right) \text {. }
$$

Any arc $k$ to be found in this section will be described as follows. On the larger base of $D^{+}$mark off $l_{+}(>0)$ collinear points $p_{i}^{+}, 1 \leqq i \leqq l_{+}$, and on the smaller base the points $q_{i}^{+}=h_{1}\left(p_{i}^{+}\right), 1 \leqq i \leqq l_{+}$. In $D^{+}$construct $l_{+}$disjoint simple polygonal arcs $K_{i}^{+}, 1 \leqq i \leqq l_{+}$, with the $p_{i}^{+}$'s and $q_{i}^{+}$'s as end points and with no other points in common with the boundary $D^{+} .\left(p_{i}^{+}\right.$and $q_{i}^{+}$are not necessarily the end points of $K_{i}^{+}$.) Let $K^{+}$be the union of the $K_{i}^{+}$'s. In the same manner in $D^{-}$construct $l_{-}(>0)$ disjoint polygonal arcs $K^{-}$with end points $p_{i}^{-}$and $q_{i}^{-}, 1 \leqq i \leqq l$. An arc $k$ is described by fitting together infinitely many copies of $K^{+}$and $K^{-}$as follows:

$$
k=\left[\bigcup_{n=1}^{\infty} h_{n}^{+}\left(K^{+}\right)\right] \cup p \cup\left[\bigcup_{n=1}^{\infty} h_{n}^{-}\left(K^{-}\right)\right],
$$

where $p$ denotes the origin $(0,0,0)$. (See Figure 4.) In the following all arcs are described simply by giving $K^{+}$in $D^{+}$and $K^{-}$in $D^{-}$. If such an arc is right or left tame, $D^{+}$or $D^{-}$is respectively omitted.

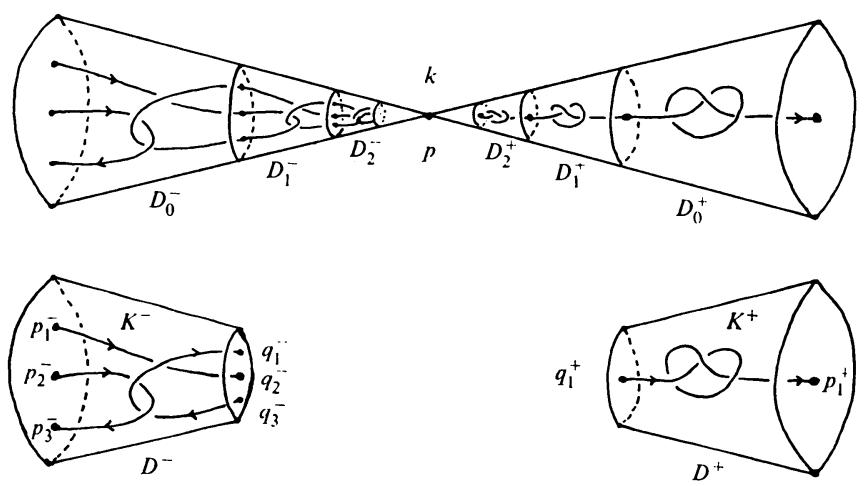

Figure 4 
Throughout the following examples, the methods of ([13, p. 981]) are used in computing the fundamental group.

EXAMPLE 1. We first consider an arc $k_{1}$ at a point $p$ which is of the trivial local type. In this case [ $Z$, identity] and $J Z$ are respectively a local $Z$-group and a local module. Thus, $\Lambda_{n}\left(k_{1}, p\right)=1$ for every $n \geqq 0$.

EXAMPLE 2. Figure 5(a). With II.B.6, II.C.6, and V.A.4 it may be shown that $\Lambda_{n}\left(k_{2}, p\right)=\left(1-t+t^{2}\right)^{\infty}$, for $n \geqq 0$. The comparing topology (V.A.5) of $k_{2}$ at $p$ is $\left(1-t+t^{2}\right)^{\infty}$.
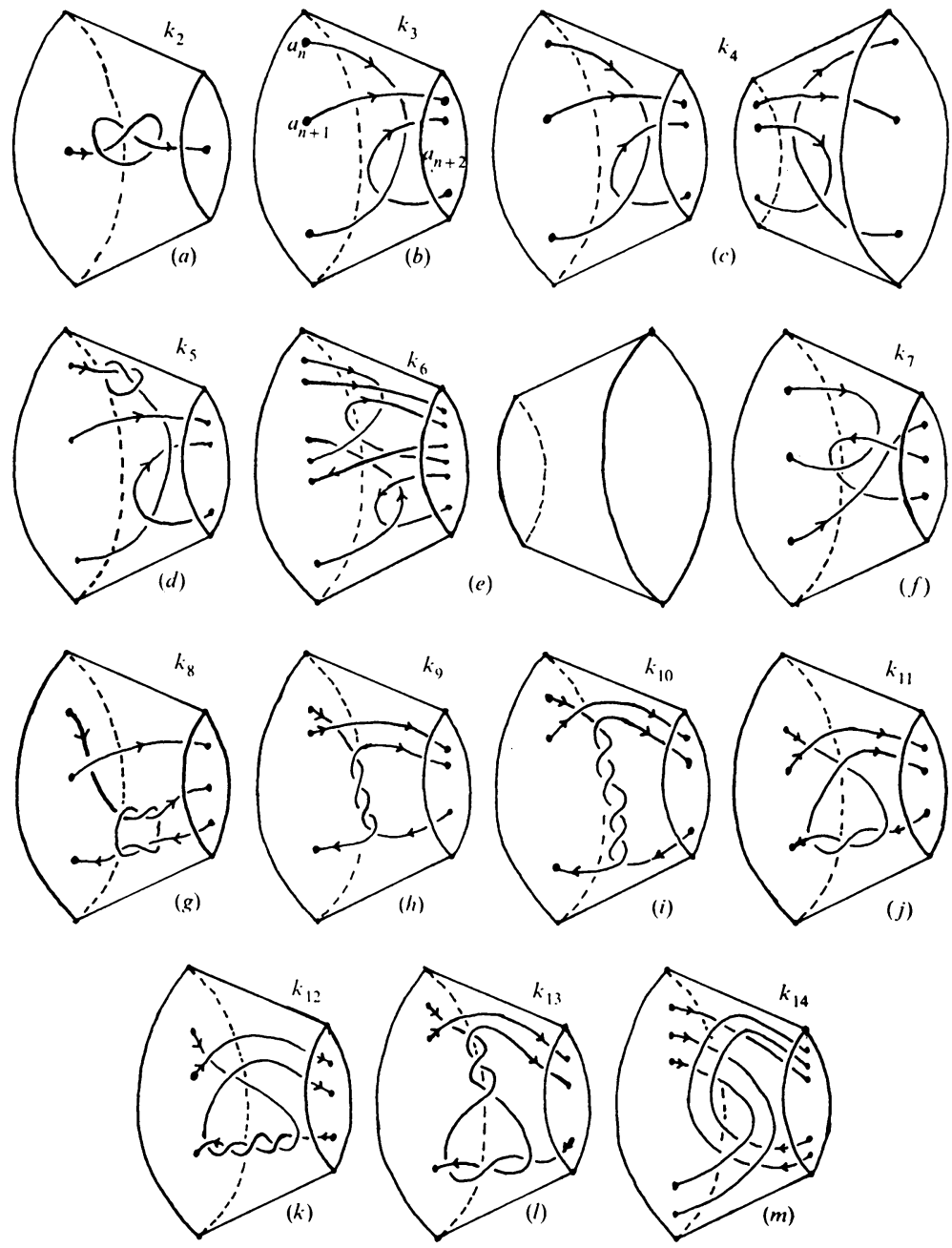

Figure 5

EXAMPLE 2'. Let $k_{2}^{\prime}$ be a Wilder arc which may be decomposed (V.A.3) into the sequence of prime tame knots $K_{1}, K_{2}, K_{3}, \ldots$ Moreover, assume that the respective Alexander polynomials $p_{1}, p_{2}, \ldots$ of these knots are relatively prime. 
Then $\Lambda_{0}(k, p)=1$. But the comparing topology (V.A.5) of $k$ at $p$ is the nontrivial topology $\prod_{n=1}^{\infty} p_{n}$.

EXAMPLE 3. Figure 5(b). A presentation of $\Pi\left(U-k_{3}\right)$ is

$$
\left(a_{0}, a_{1}, \ldots: s, r_{1}, r_{2}, \ldots\right) \text {, }
$$

where $r_{n}=a_{n+1} a_{n+2} a_{n+3} a_{n+2}^{-1} a_{n+1}^{-1} a_{n} a_{n+1} a_{n+2}^{-1} a_{n+1}^{-1} a_{n}^{-1} a_{n+1} a_{n+2} a_{n+3}^{-1} a_{n+2}^{-1}$ ( $n=0,1$, $2, \ldots)$ and $s$ is the relation about the vertex p. (See [13, p. 981].) Omitting $s$, we obtain a presentation of a locally equivalent group. The corresponding matrix is:

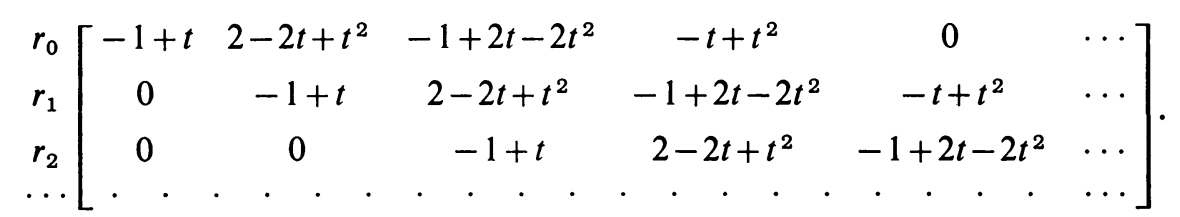

Employing II.C.6, II.A.5, II.C.5, we have $\Lambda_{0}\left(k_{3}, p\right)=(1-t)^{\infty}$ and $\Lambda_{n}\left(k_{3}, p\right)=1$, for $n \geqq 1$. Since by V.A. $6 k_{3}$ is not a Wilder arc and since $P\left(k_{3}, p\right) \leqq 4$, it follows that $P\left(k_{3}, p\right)=4$.

EXAMPLE 4. Figure 5(c). Since $k_{4}$ is an interior composite of its left and right factors $k_{3}$ and $k_{3}^{\prime}$ respectively, it follows from V.C.II that each local module of $k_{4}$ at $p$ is locally equivalent to the direct sum of local modules of $k_{3}$ and $k_{3}^{\prime}$ at $p$. Hence, by II.B.5:

$$
\Lambda_{n}\left(k_{4}, p\right)=\sum_{i+j=n}^{*} \Lambda_{i}\left(k_{3}, p\right) \Lambda_{j}\left(k_{3}^{\prime}, p\right) .
$$

Since $k_{3}^{\prime}$ at $p$ is the mirror image of $k_{3}$ at $p$, it has the same local topologies as $k_{3}$ at $p$. Thus, $\Lambda_{n}\left(k_{4}, p\right)=(1-t)^{\infty}$, for $n=0$ or 1 , and $\Lambda_{n}\left(k_{4}, p\right)=1$, for $n \geqq 2$. From V.A.6 and V.C.13, $P\left(k_{4}, p\right)=6$.

EXAMPLE 5. Figure 5(d). This is an interior composite of $k_{3}$ and $k_{2}$ at $p$. Thus, from V.C.II and II.B.5 it follows that $\Lambda_{0}\left(k_{5}, p\right)=(1-t)^{\infty}\left(1-t+t^{2}\right)^{\infty}$ and $\Lambda_{n}\left(k_{5}, p\right)$ $=\left(1-t+t^{2}\right)^{\infty}$, for $n \geqq 1$. Also from V.A.6 we have the $P\left(k_{5}, p\right)=4$.

EXAMPLE 6. Figure 5(e). Using the same methods as in Example 3, we have $\Lambda_{0}\left(k_{6}, p\right)=(1-t)^{\infty}$ and $e\left(1-t, \Lambda_{1}\left(k_{6}, p\right)\right) \geqq 3$. It is reasonable to conjecture that $\Lambda_{1}\left(k_{6}, p\right)=(1-t)^{\infty}$ and $\Lambda_{n}\left(k_{6}, p\right)=1$, for $n \geqq 2$.

EXAmple 7. Figure $5(f) . k_{7}$ at $p$ has the same invariants as $k_{3}$ at $p$.

EXAMPLE 8. Figure $5(g)$. In computing the invariants of $k_{8}$ at $p$, matrix transformations must be used to give the relation matrix the form of II.C.5. $\Lambda_{0}\left(k_{8}, p\right)$ $=\left(1-t+t^{2}\right)^{\infty}$. It appears that $\Lambda_{n}\left(k_{8}, p\right)=\left(1-t+t^{2}\right)^{\infty}, n \geqq 0$.

EXAMPLE 9. Figure $5(h) . \Lambda_{0}\left(k_{9}, p\right)=2^{\infty}(1-t)^{\infty}$ and $P\left(k_{9}, p\right)=4$.

EXAmple 10. Figure $5(i) . \Lambda_{0}\left(k_{10}, p\right)=3^{\infty}(1-t)^{\infty}$ and $P\left(k_{10}, p\right)=4$.

EXAMPLE 11. Figure $5(j) . \Lambda_{0}\left(k_{11}, p\right)=(1-t)^{\infty}\left(1+t^{2}\right)^{\infty}$ and $P\left(k_{11}, p\right)=4$.

EXAmple 12. Figure $5(k) . \Lambda_{0}\left(k_{12}, p\right)=(1-t)^{\infty}\left(1+t^{2}+t^{4}\right)^{\infty}$ and $P\left(k_{12}, p\right)=4$.

EXAmple 13. Figure $5(l) . \Lambda_{0}\left(k_{13}, p\right)=(1-t)^{\infty}\left(2-t+2 t^{2}\right)^{\infty}$ and $P\left(k_{13}, p\right)=4$.

EXAMPLE 14. Figure $5(m) . \Lambda_{0}\left(k_{14}, p\right)=(1-t)^{\infty}\left(1+t^{4}+t^{5}\right)^{\infty}$. The lack of symmetry in $\Lambda_{0}\left(k_{14}, p\right)$ can easily be seen to imply that the arc $k_{14}$ is noninvertible. 


\title{
REFERENCES
}

1. W. R. Alford and B. J. Ball, Some almost polyhedral wild arcs, Duke J. Math. 30 (1963), 33-38.

2. B. J. Ball, Penetration indices and applications, Topology of 3-manifolds and related topics, Proc. Univ. Georgia Inst., pp. 37-39, Prentice-Hall, Englewood Cliffs, N. J., 1962.

3. R. H. Bing, An alternate proof that 3-manifolds can be triangulated, Ann. of Math. 69 (1959), 37-65.

4. - Locally tame sets are tame, Ann. of Math. 59 (1954), 145-158.

5. R. C. Blanchfield, Intersection theory of manifolds with operators, Ann. of Math. 65 (1957), 340-356.

6. E. J. Brody, On infinitely generated modules, Quart. J. Oxford 11 (1960), 141-150.

7. R. H. Crowell, On the Van Kampen theorem, Pacific J. Math. 9 (1959), 43-50.

8. R. H. Crowell and R. H. Fox, Introduction to knot theory, Ginn, Boston, Mass., 1963.

9. R. H. Fox, A remarkable simple closed curve, Ann. of Math. 50 (1949), 264-265.

10. - Free differential calculus. I, Ann. of Math. 57 (1953), 547-560.

11. - Free differential calculus. II, Ann. of Math. 59 (1954), 196-210.

12. - Free differential calculus. V, Ann. of Math. 71 (1960), 408-422.

13. R. H. Fox and E. Artin, Some wild cells and spheres in three-dimensional space, Ann. of Math. 49 (1948), 979-990.

14. R. H. Fox and O. G. Harrold, The Wilder arcs, Topology of 3-manifolds and related topics, Proc. Univ. Georgia Inst., pp. 184-187, Prentice-Hall, Englewood Cliffs, N. J., 1962.

15. W. Graeub, Die Semilineare Abbildungen, S.-B. Heidelberger Akad. Wiss. Math.-Natur. K1. 1950 (1950), 205-272.

16. W. Hurewicz and H. Wallman, Dimension theory, Princeton Univ. Press, Princeton, N. J., 1948.

17. A. G. Kurosh, The theory of groups, Vol. II, Chelsea, New York, 1960.

18. S. Lefschetz, Algebraic topology, Colloq. Publ., Vol. 27, Amer. Math. Soc., Providence, R. I., 1942.

19. E. E. Moise, Affine structures in 3-manifolds. VII, Ann. of Math. 58 (1953), 403-408.

20. L. P. Neuwirth, Knot groups, Princeton Univ. Press, Princeton, N. J., 1965.

21. H. Seitfert and W. T. Threlfall, Lehrbuch der Topologie, Chelsea, New York, 1947.

22. H. Schubert, Knoten and Vollringe, Acta. Math. 90 (1953), 131-286.

\author{
Princeton University, \\ Princeton, New Jersey \\ FLoRida STATE UNIVERSity, \\ Tallahassee, Florida
}

